In Building the European Capacity in Operational Oceanography. Proceedings of the Third International Conference on EuroGOOS 36 December 2002 - Athens, Greece. pp 146-153 2003

Eds H. Dahlin, N.C. Flemming, K. Nittis, S.E. Petersson

ISBN 9780444515506

https://doi.org/10.1016/S0422-9894(03)80025-9

https://archimer.ifremer.fr/doc/00706/81841/

\title{
Oceanpal: an instrument for remote sensing of the ocean and other water surfaces using GNSS reflections
}

\author{
Ruffini $\mathrm{G}^{1,}{ }^{*}$, Caparrini $\mathrm{M}^{1}$, Chapron Bertrand ${ }^{2}$, Soulat $\mathrm{F}^{1}$, Germain $\mathrm{O}^{1}$, Ruffini $\mathrm{L}^{1}$ \\ ${ }^{1}$ Starlab, Edifici de l'Observatori Fahra, Spain \\ 2 IFREMER, Ctr Brest, Brest, France. \\ * Corresponding author : G. Ruffini, email address : giulio.ruffini@starlab.es
}

\begin{abstract}
:
This paper describes Oceanpal, an inexpensive, all-weather, passive instrument concept for remote sensing of the ocean and other water surfaces. Oceanpal is based on the use of reflected signals emitted from GNSS, and as such it is well grounded on the growing, long term GNSS infrastructure. As seen from the instrument, several GNSS emitters are simultaneously in view at any given time, providing separated multiple scattering points with different geometries. Reflected signals are affected by surface "roughness" and motion (i.e. sea state, orbital motion, and currents), mean surface height and dielectric properties (i.e. salinity and pollution). Oceanpal is envisaged to act as an accurate, "dry" tide gauge/surface monitoring system as a part of a future distributed ocean remote sensing network concept.
\end{abstract}

Keywords : GNSS-R, altimetry, remote sensing, GPS, Galileo 


\section{Introduction}

Two GNSS constellations are presently operational, the Global Positioning System (GPS), owned by the United States, and the Russian GLObal NAvigation Satellite System (GLONASS). In the next few years, the European Satellite Navigation System (Galileo) will be deployed. By the time Galileo becomes operational in 2008, more than 50 GNSS satellites will be emitting L-band spread spectrum signals with a well characterized structure, and they will remain in operation for at least a few decades. These signals can be used within the GCOS/GOOS. The immediate objective of the Starlab Oceanpal project is the development of technologies for operational in-situ or low-altitude water surface monitoring using GNSS Reflections, a passive, all weather radar technology of great potential.

Oceanpal is an offspring of technology developed within several ESA/ESTEC projects targeted on the exploitation of GNSS Reflections from space ${ }^{1}$, an example of bistatic 
(passive) radar (Cantafio 1989). Although our focus here is on low altitude applications, it is worthwhile explaining in more detail the rationale for spaceborne deployment: an important aspect of the GNSS-R concept is the synergy between space and ground monitoring using the same technology and the same signal infrastructure, which will ensure homogeneity in the measurements.

\section{GNSS-R in space: the Petrel Earth Explorer concept}

In the future, the artificial separation between geophysical "layers" (ocean, troposphere, stratosphere, ...) will disappear. In particular, future models will need to reflect the fundamental role of atmosphere-ocean coupling. The sea surface provides the oceanatmosphere link, regulating momentum, energy and gas exchange, and several fundamental ocean circulation features are directly related to wind-wave induced turbulent transports in the oceanic mixed layer. Vertical transfers of energy and momentum are associated to wave breaking: wave breaking controls the rate of turbulent mixing in the upper ocean layer and is directly related to surface roughness. Upper temperature structure and inertial currents are strongly modified during strong meteorological forcing and at mesoscales such modification will impact eddy dynamics. Eddies and gyres are important to climate models and are fundamental to understand mixing, heat transport and feedback to general circulation, as well as transport of nutrients, chemicals and biota for biochemical processes.

At the atmosphere-ocean boundary, many temporal and spatial scales play an important role: from the molecular to the synoptic level, from seconds to eons. For this reason, observing this surface appropriately is an important challenge for global observation systems, which will require high resolution, large swathes, frequent revisits and longterm stability (Le Traon 2002). All of these are part of the GNSS-R concept.

The ocean-atmosphere interface is characterized (to the lowest statistical order) by the geophysical variables of local mean sea level $(h)$, significant wave height $(s w h)$ and directional mean square slope (dmss). Mesoscale measurements of sea surface dmss are an important missing element from the Global Climate and Ocean Observation Systems, and are needed to understand and quantify the atmosphere-ocean flux of energy, momentum and gas. Moreover, since ocean forcing is a strongly intermittent (in both space and time) non-linear phenomenon, frequent space-time collocated mesoscale measurements of $h$ and of $d m s s$ are also needed. The scientific objectives of a spaceborne GNSS-R mission (the Petrel concept, recently submitted to the Earth Explorer ESA program) therefore the medium and long-term components for Physical Climate Observation (Theme 2 of the Earth Explorer Program) with a focus on providing a key element for the study of atmosphere-ocean coupling. The elementary geophysical products such a mission would provide are indeed mesoscale collocated altimetric and sea surface directional mean square slope measurements. These measurements are also of great interest for the observation of surface winds, mean sea surface, sea-ice, ionospheric electron content and salinity.

\footnotetext{
${ }^{1}$ Such as the ESA/ESTEC projects OPPSCAT, OPPSCAT 2 (Speculometry), Paris-Alpha, ParisBeta, Paris-Gamma (altimetry) and GIOS-1 (focusing on ionospheric monitoring).
} 
Results from recent ESA studies and experiments and also from work in the US indicate that GNSS-R data can provide sufficient information to resolve mesoscale features in the ocean, as well as collocated directional mean square slope measurements (for further information, the reader is directed to the references). In Figure 1 we can see a schematic rendition of a spaceborne GNSS-R mission, as well as an illustration showing multiple (GPS) reflections available to a ground receiver during a 24-hour period. Note the multi-static character of the technique: a single passive instrument can provide a large swath, thanks to the availability of multiple emitters.
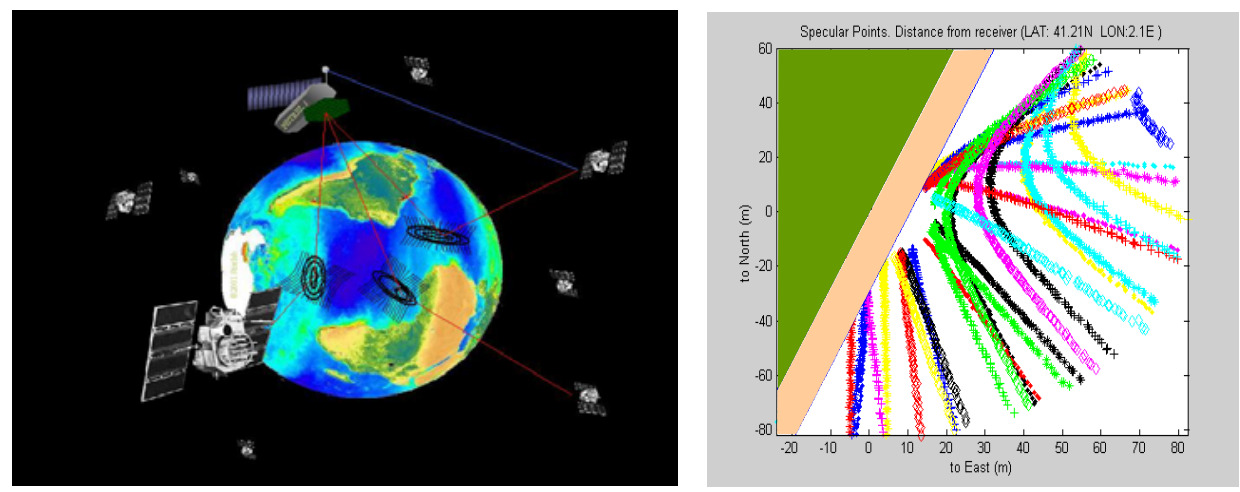

Figure 1: Left: Artist concept of the bistatic GNSS spaceborne concept. GNSS signals reflect off the Earth surface and are gathered by a spaceborne receiver. All direct signal links are not shown for simplicity. Right: GPS-R specular points after 24 hours as seen from a receiver at $50 \mathrm{~m}$ altitude in the Barcelona coast. A conservative cut-off of 20 degrees in the local elevation of the reflected signals has been used for display purposes.

\section{The Oceanpal concept}

Starlab is developing an operational instrument based on GNSS-R. Initial results indicate that this sensor can provide very useful altimetry and sea state information from, at least, low altitude applications (e.g., coasts or aircraft). The company is perfecting robust algorithms for operational code and phase tracking of the reflected field and extraction of geophysical parameters. Reflected signals carry significant information on sea state and topography, and experimental work as well as simulations have demonstrated the potential of this concept for coastal (centimetric) altimetry and sea state monitoring. The principle of operation is simple (Figure 2): the instrument receives the direct and reflected GNSS signal from a given satellite. Due to the longer path, the reflected signal takes a bit longer to arrive: this effect provides the basis for ranging and altimetry, exploiting the centimetric precision of GNSS technology. As seen from the instrument, several GNSS emitters are simultaneously in view at any given time, providing information from separated scattering points with different geometries and thus strengthening the extraction of oceanographic variables. Reflected signals are affected by surface "roughness" and motion (sea state, orbital motion, currents), mean surface height and surface dielectric properties (i.e., salinity and pollution). The instrument characterizes the "noisy" reflected electric field to infer ocean, river, or lake surface properties, using robust techniques. 


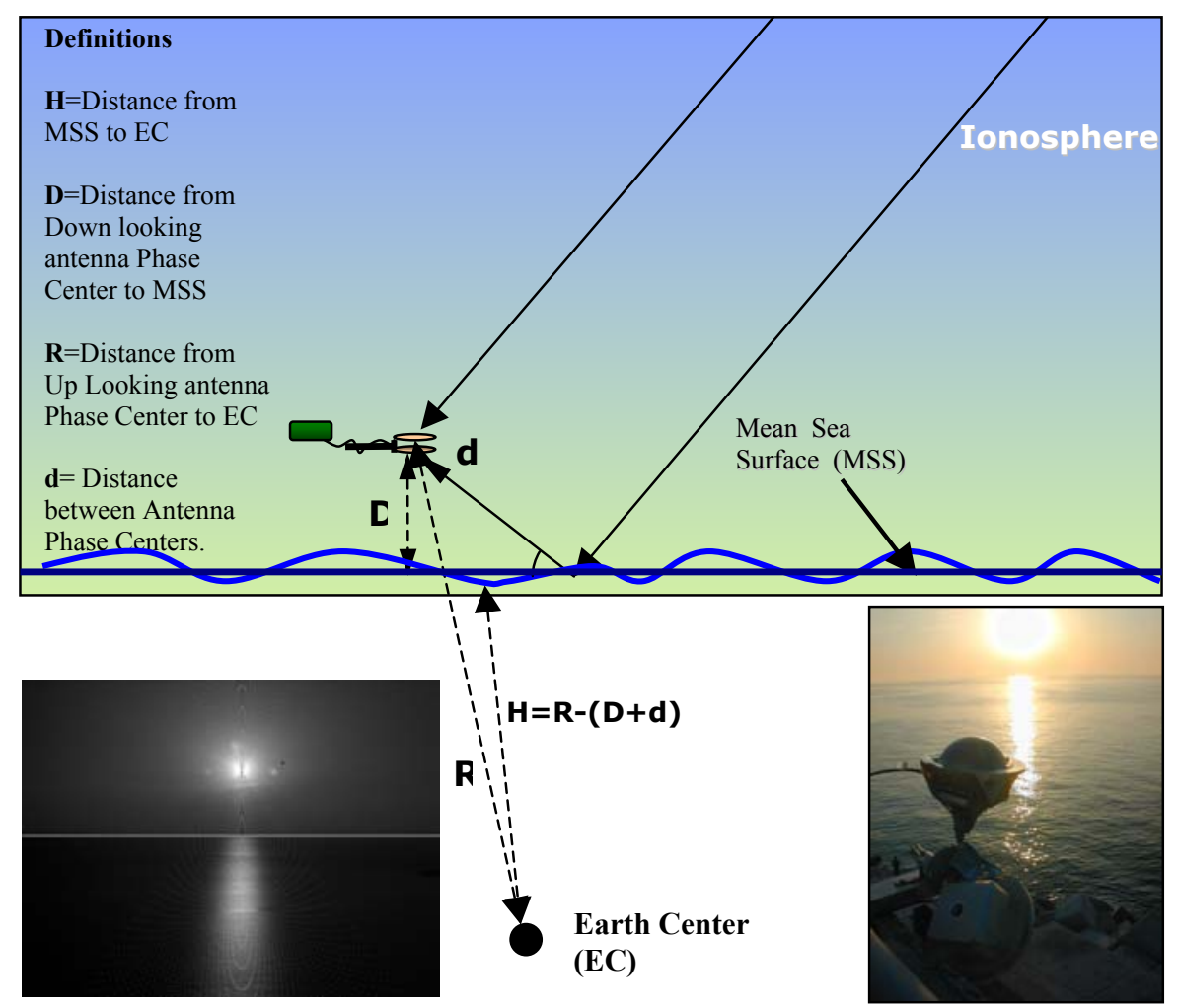

Figure 2: Simplified schematic representation of the opportunistic bistatic GNSS-R concept. The direct and reflected signals originating from a GNSS source are combined at the receiver to estimate the distance to the surface and to the Earth Center (atmospheric errors cancel out for a low altitude receiver). On the bottom right, a detail of an early Coastpal Experiment in the Barcelona Port using equipment provided by ESA-ESTEC. The up and down looking antennas can be seen. On the bottom, left, example of analysis of optical glitter to support GNSS-R analysis. Slope statistics can be calculated using optical measurements.

Although bistatic radar can work using various sources of opportunity, GNSS are in many ways unique: Oceanpal GNSS-R altimetric products are very stable, long-term and can provide, automatically, absolutely calibrated mean sea level. Thanks to its GNSS heritage, Oceanpal is an inexpensive, all-weather, dry and passive concept for remote sensing of the ocean and other water surfaces, for accurate provision of sea state and altimetry. The instrument can be deployed on multiple platforms: static (coasts, harbors, off-shore), and slowly moving (e.g., boats, floating platforms, buoys, stratospheric platforms, aircraft, ...). Spaceborne application requires further technology development, and is the subject of several ongoing ESA projects.

We envision that this system will act as an accurate, distributed, "dry" tide gauge network while providing other surface monitoring, providing a stable and precise service based on the growing long term GNSS infrastructure. As such, Oceanpal is part of another Starlab vision in which small, multiple inexpensive sensors will exchange information to "synthesize" an extended remote sensing system and provide relevant 
oceanographic information to a whole array of end-users (GOOS, Public Authorities, harbours, shipping, fishing industry, off-shore mining, and in general to those conducting their activities in or near the sea).

\section{Recent coastal experimental campaigns}

Several experiments have taken place to date: from space, stratospheric balloons, aircraft and the ground. Here we shall focus on the latter (see the references for further information).

Recent coastal GNSS-R experimental campaigns led by Starlab have collected data from low altitude with a wide range of sea state conditions, mostly using experimental equipment lent by ESA/ESTEC. Some of these campaigns have been carried with the logistic support of the Barcelona Port Authority.

As shown in Figure 2, two antennas are usually employed to collect GPS signals: one antenna is zenith looking and Right Hand Circularly Polarized to gather the direct GPS signal, while the other is nadir/side looking and Left Hand Circularly Polarized to recover the reflected signal. The output from each the antenna is sent to a GPS receiver. The IF data generated by the receivers is then recorded at a sufficiently high sampling frequency, after (typically) being digitalized at one bit. The experimental data has been fed to Starlab's GPS-Reflections processor (STARLIGHT ${ }^{2}$ ), which is used to retrieve the reflected electromagnetic field and estimate sea level and sea state. The processor, through the conventional correlation method, evaluates the reflected field magnitude and phase. The retrieved field, though somehow distorted by processing, retains very useful information about the characteristics of the reflecting surface. Comparison between this field and the direct one is then performed to infer the desired quantities, such as sea roughness and sea level. Recent altimetric results are at the centimetre level, and there is very good correlation between sea state and field dynamics.

In Figure 2 a detail of the experimental hardware setup is shown. This particular experiment took place at dawn. Along with the GPS signals another source of opportunity was exploited: the Mediterranean rising sun. The use of multifrequency bistatic specular scattering instruments is very important to validate models, and may provide clues on how separate ocean surface spectral parameters (such as surface wind and wave age).

As seen from a static platform, the electric field scattering from a frozen ocean could be represented as a static phasor. The motion of the ocean surface translates into motion of the phasor. In Figure 3 we can see a phasor simulated using Fresnel scattering from an ocean generated using the Elfouhaily et al. (1997) ocean spectrum, and the real thing. Analysis of the dynamics of the reflected phasor provides the key to estimating sea surface parameters from such static platforms. Aircraft of spacecraft observations must be analysed differently, basically exploiting the size and shape of the "glistening" zone (as in Spooner 1822). The fundamental tool to study this is provided by the Delay Doppler mapping capability of GNSS-R (see, e.g., Ruffini 1999, Ruffini 2000a).

\footnotetext{
${ }^{2}$ STARLab Interferometric GNSS Toolkit.
} 

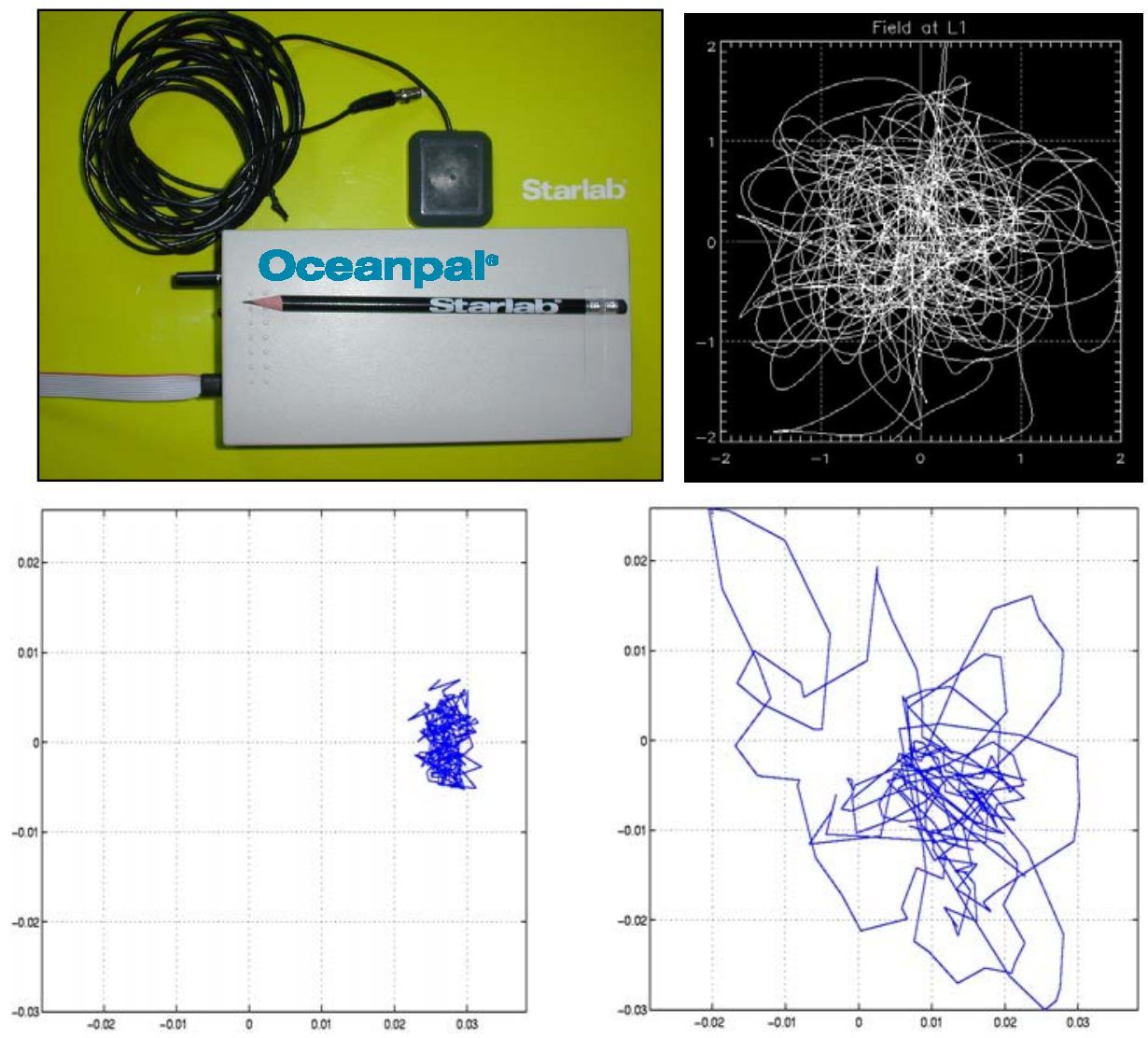

Figure 3. Top, left: Starlab's Oceanpal prototype is a GNSS-R sensor ideally suited for coastal, river or lake applications. Top right: The dynamic L-band reflected electric field phasor from a virtual moving ocean after a few seconds of time evolution, from a simulation using Starlab's GRADAS software package Bottom: The dynamic phasor of the direct (left) and reflected (right) GPS L1 field after a few seconds of time evolution, from a coastal experiment processed using Starlab's STARLIGHT software.

\section{Acknowledgements}

Parts of this work were carried out under GNSS-R ESA/ESTEC contracts: OPPSCAT (13461/99/NL/GD), OPPSCAT 2 (3-10120/01/NL/SF) as well as ESA/ESTEC Contract 15083/01/NL/MM (Paris Beta), ESA/ESTEC Contract No. 14285/85/nl/pb, Starlab CCN3-WP3 (Paris Alpha). Many thanks also to the Barcelona Port Authority for experimental logistics support and to our partners in ESA projects.

\section{References}

Cantafio, L.J., "Space-based Radar Handbook”, 1989, Artech House, 1989.

Caparrini, M., 1998, Using reflected GNSS signals to estimate surface features over wide ocean areas. ESTEC Working Paper No. 2003, Dec 1998. 
Cardellach, E., G. Ruffini, D. Pino, A. Rius, A. Komjathy MEditerranean Balloon EXperiment: GPS reflections for wind speed retrieval from the stratosphere. Remote Sensing and Environment, 2002.

Elfouhaily, T., Chapron, B., Katsaros, K., Vandemark, D., 1997, A unified directional spectrum for long and short wind-driven waves, J. Geoph. Res., vol 102, no. C7, p. 15,781--1,796, July 15, 1997.

Garrison, J.L, G. Ruffini, A. Rius, E. Cardellach, D. Masters, M. Armatys, V.U Zavorotny, 2000, Preliminary results from the GPSR Mediterranean Balloon Experiment (GPSR-MEBEX), Proceedings of ERIM 2000, Remote Sensing for Marine and Coastal Environments, Charleston, 1-3 May, ISSN 1066-3711, 2000.

Le Traon, P.Y., G. Dibarboure, G. Ruffini, E. Cardellach, 2002, Mesoscale Ocean Altimetry Requirements and Impact of GPS-R measurements for Ocean Mesoscale Circulation Mapping, Abridged Starlab ESA/ESTEC Technical Report from the Paris Beta project, ESA/ESTEC Contract 15083/01/NL/MM, Courtesy of ESA/ESTEC and Starlab, http://arxiv.org/abs/physics/0212068, Dec 2002.

Lowe, Stephen T.; LaBrecque, John L.; Zuffada, Cinzia; Romans, Larry J.; Young, Larry E.; Hajj, George A., 2002, First spaceborne observation of an Earth-reflected GPS signal, Radio Science, Vol. 37, No. 1, 07 February 2002.

Martín -Neira, M., 1993, A passive reflectometry and interferometry system (PARIS): application to ocean altimetry, ESA Journal, vol 17, pp 331-355, 1993.

Martín -Neira, M., Colmenarejo, P., Ruffini, G., Serra, 2000, C., Ocean altimetry using the carrier phase of GNSS reflected signals", paper presented at Ocean Winds 2000 workshop at IFREMER.

Martín-Neira, M., Caparrini, M.,Font-Rossello, J., Lannelongue, S. and Serra Vallmitjana, C., 2001, The PARIS Concept: an Experimental Demonstration of Sea Surface Altimetry Using Reflected GPS Signals, IEEE Trans. Geoscience and Remote Sensing, vol. 39, no. 1, 2001

Ruffini, G, Cardellach, E., Rius, A., Aparicio, J.M., 1999, GNSS-OPPSCAT WP1000 ESA Report: Remote Sensing of the Ocean by Bistatic Radar Observations: a Review. By Courtesy of ESTEC (GNSS-OPPSCAT project, ESTEC Contract 13461/99/NL/GD), available at http://217.126.65.140/library/WP1000.ps.gz

Ruffini, G., J.L. Garrison, E. Cardellach, A. Rius, M. Armatys, D. Masters, 2000a, Inversion of GPSR Delay-Doppler Mapping Waveforms for wind retrieval, IGARSS, Honolulu, July 2000. This document can be found at http://217.126.65.140/staff/giuliospapers/igarss2000.ps.gz.

Ruffini, G., Soulat, F., 2000b, Paris Interferometric Processor Theoretical Feasibility Study part, ESA's PIPAER-IEEC-TN-1100/2100, ESTEC Contract No. 14071/99/NL/MM. Courtesy of ESA: http://arxiv.org/ps/physics/0011027

Ruffini, G., Marco Caparrini, Leonardo Ruffini, 2002, PARIS Altimetry with L1 Frequency Data from the Bridge 2 Experiment, Abridged Starlab ESA/ESTEC Technical Report from the Paris Alpha CCN3, Report-no: ESA/ESTEC Contract No. 14285/85/nl/pb, Starlab CCN3-WP3. http://arxiv.org/abs/physics/0212055, Dec 2002.

Spooner, J.,1822, Sur la lumiere des ondes de la mer, Corresp. Astronomique du Baron de Zach, 6:331, 1822.

Zavorotny, V.U., Voronovich, A.G., 2000, Scattering of GPS Signals from the Ocean with Wind Remote Sensing Application, IEEE Transactions on Geoscience and Remote Sensing, Vol. 38, No. 2, pp. 951-964. 